East African Medical Journal Vol. 81 No. 7 July 2004

PRIMARY BREAST SARCOMA: CASE REPORT

S. Hassan, BSc., MBChB, MMed, (Surg) FCS, Consultant Surgeon, Nairobi Womens Hospital Breast Center and Senior Lecturer, Department of Human Anatomy, College of Health Sciences, University of Nairobi, P.O. Box 30197, Nairobi, Kenya and G. Adari, MBChB., MMed(Surg) Consultant Surgeon, Nairobi, Womens Hospital

Request for reprints to: Dr. S. Hassan, Department of Human Anatomy, College of Health Sciences, University of Nairobi, P.O. Box 30197, Nairobi, Kenya

\title{
PRIMARY BREAST SARCOMA: CASE REPORT
}

\author{
S. HASSAN and G. ADARI
}

\begin{abstract}
SUMMARY
Primary breast sarcoma is a rare entity occurring in $0.5 \%$ of women with breast malignancy. Like in breast carcinoma, delay in its diagnosis has important clinical and treatment implications. The subject of this report presented at our breast unit with advanced breast lesion months after she noticed a small lump in her right breast. She had no clear diagnosis despite several consultations, in-patient treatments at two facilities in the city, breast ultrasonography, breast mammography and three fine needle aspiration cytology (FNAC) examinations. The patient needed multiple blood transfusions. A final FNAC showed ductal carcinoma. Histology following wide excision confirmed high-grade primary stromal breast sarcoma. She required adjuvant combination chemotherapy. A combination of diagnostic failures and patient fault caused delay in subject's treatment. Lesion progression during delay which influenced the pattern of physical morbidity, tumour prognosis and need for adjuvant treatment. Embracing the concept of breast care in dedicated breast units may minimise such treatment delays.
\end{abstract}

\section{INTRODUCTION}

Primary breast sarcoma (PBS), first described in 1887 , occurs in less than $1 \%$ of women with breast malignancy $(1,2)$. The entity, which arises from breast mesenchyme, includes angiosarcoma, malignant fibrous histiocytoma, stromal sarcoma, liposarcoma, leiomyosarcoma, dermatofibrosarcoma protuberans, osteosarcoma, fibrosarcoma and rhabdomysarcoma(2). Although often confused with cystosarcoma phyllioides, phylloid sarcomas comprise mixtures of epithelial and mesenchymal elements(3). Nonetheless, distinguishing them is academic as their clinical courses and survival rates are similar(4). The optimal treatment for breast sarcoma is still unclear. There is no consensus on the extent of surgery, and the need for adjuvant chemoirradiation. Some studies on radiotherapy and chemotherapy depict effect on local recurrences and survival respectively(5-8). Overall, the prognosis of breast sarcoma is poor. Like common soft tissue sarcomas, tumour grade and size of the lesion influence prognosis. Early diagnosis and treatment impact on survival. This report traces the evolution of a breast lesion during a long period of diagnosis and highlights the clinical and prognostic effects of the delay.

\section{CASE REPORT}

F.A. was a 46 year-old para $3+0$ peri-menopausal and educated woman whose last delivery was 17 years earlier. She discovered a painless lump in her right breast and urgently sought a surgical opinion. Aspiration of the lump revealed malignant cells and mastectomy was advised. Opting for a second opinion she presented at a Nairobi hospital where she was admitted due to associated anaemia. Here, she received compatible blood and had two further FNAC examinations. Malignant cells were not observed in both examinations. A mammogram showed a well-circumscribed benign lesion (Figure 1). Two weeks later, she left the hospital before a firm diagnosis could be established. She consulted another physician who ordered repeat images at a different hospital. The images were negative for malignancy. She nursed her lesion at home until three months later when it ulcerated. F.A. sought alternative therapy for the now heavy, infected, foul smelling and easily bleeding swelling. Two weeks of herbal treatment during this admission did not alleviate her symptoms. Her general state deteriorated and the breast wound became more offensive.

We received a para $3+0$ lady whose last delivery was 17 years earlier. She was sick looking and markedly pale. She had no lymphadenopathy. Her chest was clear and heart sounds normal. On local examination, she had a huge and foul-smelling fungating mass in the upper outer quadrant of the right breast (Figure 2). It was $10 \times 15 \mathrm{~cm}$, pedunculated, mobile and nontender. The axillary nodes were not involved. We made an impression of T4NOM0 carcinoma of breast to rule out phylloides sarcoma.

The chest radiograph and abdominal scan were normal. Her haemoglobin was $5.8 \mathrm{~g} / \mathrm{dl}$. She received 4 
units of compatible blood and the daily wound cleaning. Repeat FNAC results showed sheets of loose malignant ductal cells, a background of tumour diathesis, mitotic figures and tumour giant cells. We optimized her for wide resection and delayed wound closure. Her haemoglobin peaked at $9.9 \mathrm{~g} / \mathrm{dl}$. The platelets were adequate as was the renal function. Other screening results were also normal. A KUB ultrasound was normal save for many fibroids.

Figure 1

Mammogram of the right breast showing a well defined rounded capacity about $5 \mathrm{~cm}$ in diameter with a benign fatty halo. No radiological evidence of malignancy is seen

\section{Figure 2}

The right breast with a $15 \mathrm{~cm}$ in diameter, ulcerated mass

Figure 3

The right breast showing a healed mastectomy scar
Early in the 5th month, we undertook wide resection. The $700 \mathrm{gm}, 150 \mathrm{~mm}$ pedunculated mass was mucoid to cut and exhibited haemorrhagic necrosis on section. Histology showed complete excision of a high grade stromal breast sarcoma. Sections showed malignant spindle cell tumour exhibiting marked pleomorphism with a number of giant cells and frequent mitotic bodies. Lying among the tumour cells were also benign looking multi-nucleated cells. The base of the tumour showed normal breast lobules with a round inflammatory cell infiltrate. Subsequent dressings resulted in a granulating wound which was closed secondarily. The wound edges and floor had no residual tumour. F.A.'s wound healed (Figure 3), her haemoglobin normalised $(12.0 \mathrm{~g} / \mathrm{dl})$ and was discharged to start on a 6-cycle regime of vincristine $1.4 \mathrm{mg} / \mathrm{m}^{2}$, adriamycin $40 \mathrm{mg} / \mathrm{m}^{2}$, endoxan $600 \mathrm{mg} / \mathrm{m}^{2}$ every 21 days.

\section{DISCUSSION}

Delays in breast cancer diagnosis are associated with negative clinical effects. At the Edinburgh breast unit, delays occurred in $1.4 \%$ of women between 19881999. The main causes were protocol violations, clinical errors, radiological errors, pathological errors and patient fault(9). Outside dedicated breast units, delay frequencies are decidedly higher. There is no evidence of protocol violation in the current report. Delay was caused by inappropriate treatment outside mainstream care pathway, delay in appropriate investigation, lesion not identified radiologically and benign FNA from malignant lesion. Patient fault significantly contributed to the delay. The choices she made were not informed, underscoring the need for patient education. It is difficult to discern the possible role of herbal therapy in this situation. At the breast center, she was provided with detailed information of our understanding of her condition and she proved to be no barrier to her care.

Fine needle aspiration does not always sample a lesion adequately. Even when targeted accurately and sampled, interpretation may be difficult. To reduce geographical misses during aspiration and improve pathological interpretation, experienced aspirators and pathologists are respectively key $(10,11)$.

A lesion was visualized radiologically but interpreted as benign. This presents one of few situations of benign appearance of a malignant lesion. An early core sample was indicated. Some lesions evolve so that the final biopsy result could represent a new lesion. This was unlikely in F.A.'s situation as the first aspirate had shown malignant cells.

Our patient underwent breast conservation surgery without axillary dissection because we were not convinced she had breast carcinoma. She had no regional nodes despite the advanced lesion. Lymphatic spread is common in carcinoma but rare in sarcoma. 
In only one of 39 axillary dissections in a French study and two of 22 dissections at Mayo clinic $(1,2)$ were nodes histologically involved. Mastectomy is not superior to wide resection in sarcoma if the margins are clear. Recurrence rates and overall survival are not affected by extent of surgery(2). Further, majority of stromal tumours of the breast are not multicentric.

The factors that determine survival and disease recurrence are still unclear. Although tumour size and histologic grade are clearly important, prognostic variables in sarcoma in general, the reported correlation in breast is not uniform. The French study indicates worse outcome. In the Mayo series however, size of tumour and histopathology did not influence outcome. The role of adjuvant chemotherapy or radiation is also unclear. Some authors have suggested radiotherapy due to high rate of chest recurrences. Firm conclusions about the use of chemotherapy are still awaited. But if PBS behaves like other sarcomas, adjuvant chemotherapy would seem to be justified in high grade tumours measuring more than $5 \mathrm{~cm}(1)$.

In conclusion a combination of patient and diagnostic errors caused delay in the current treatment. This influenced the morbidity profile (anaemia, weakened state, ulceration, need for chemotherapy). Her treatment was spread over several centers. Care in a dedicated breast unit may minimise such delays. Current evidence does not provide a clear treatment pathway for PBS. Wide resection without axillary dissection is a surgical option in large size PBS.

\section{ACKNOWLEDGEMENTS}

To Dr. E. Njuguna for oncology opinion, Drs. K. Radia and L. Muchiri for opinion on histopathology, staff of the breast centre for taking care of the patient and Dr. P. Mardeta for reading the manuscript.

\section{REFERENCES}

1. Zelek, L., Llombart-Cussac, A., Terrier, P., et al. Prognostic factors in primaary breast sarcomas: A series of patients with long-term follow-up J. Clin. Oncology. 2003; 21:2583-2588.

2. Blanchard, D.K., Reynolds, C.A., Grant, C.S. and Donohue, J.H. Primary non phylloides breast sarcomas. Amer. J. Surg. 2003; 186:359-361.

3. Petrek, J.A. Phylloide tumors, in Harris, J.R., Lippman, S., Morrow M. et al. (eds). Diseases of the breast, Philadelphia, PA, Lippincott-Raven, 2000; pp 863-869.

4. Terrier, P.H., Terrier-Lacombe M.J., Mouriese H. et al: Primary breast sarcomas: A Review of 33 cases with immunohistochemistry and prognostic factors. Breast Cancer Res. Treat. 1989; 13:39-48.

5. Yang, J.C., Chang, A.E., Baker, A.R. et al. Randomized prospective study of the benefit of adjuvant radiation therapy in the treatment of soft tissue sarcomas of the extremity. J. Clin. Oncol. 1998; 16:197-203.

6. Pisters, P.W., Harrison, L.B., Leung, D.H., et al. Long-term results of a prospective randomized trial of adjuvant brachytherapy in soft tissue sarcoma. J. Clin. Oncol 1996; 14:859-868.

7. Rosenberg, S., Tepper, J., Galtein, E., et al. The treatment of soft-tissue sarcoma of the extremities: Prospective randomized evaluation of (1) limb-sparing surgery plus radiation therapy compared with amputation and (2) the role of adjuvant chemotherapy. Ann. Surg. 1982; 196:305-315.

8. Frustaci, S., Gherlinzoni, F., De Paoli, A., et al. Adjuvant chemotherapy for adult soft tissue sarcomas of the extremities and girdles: Results of the Italian randomized cooperative trial. J. Clin. Oncol. 2001; 19:1238-1247.

9. Barber M.D., Kack W. and Dixon J.M. Diagnostic delay in breast cancer Brit. J Surg. 2004; 91:49-53.

10. Dixon, J.M., Andersson, T.J., Lamb, J., Nixon, S.J. and Forrest, A.P.M. Fine needle aspiration cytology, in relationships to clinical examination and mammography in the diagnosis of solid breast mass. Brit. J. Surg. 1984; 71:593-596.

11. Barrows, G.H., Andersson, T.J., Lamb, J.L. and Dixon, J.M. Fine-needle aspiration of breast cancer. Relationship of clinical factors to cytology results in 689 primary malignancies. Cancer 1986; 58:1493-1498. 